\title{
Tableaux for Maximum Satisfiability in Lukasiewicz Logic
}

\author{
Chu Min Li \\ MIS, Université de Picardie \\ Amiens, France
}

\author{
Felip Manyà \\ IIIA-CSIC \\ Bellaterra, Spain
}

\author{
Amanda Vidal \\ Czech Acad Sci, Inst Comp Sci \\ Prague, Czech Republic
}

\begin{abstract}
We define a tableau calculus for solving the MaxSAT problem of 3-valued Lukasiewicz logic, and prove its soundness and completeness. The calculus can be naturally extended to other finitely-valued logics. Our contributions establish the foundations of a generic problem solving paradigm for combinatorial optimization based on Lukasiewicz logic.
\end{abstract}

\section{INTRODUCTION}

Over the last decade, there has been tremendous progress in theoretical and applied aspects of the MaxSAT problem. As a result, there are now a number of competitive solvers that are able to solve challenging optimization problems (see e.g. [1], [2], [3], [5], [6], [8], [12], [18], [20], [21], [25], [28] and the references therein for previous and related work).

From a problem solving perspective, MaxSAT is seen as a competitive generic problem solving formalisms for combinatorial optimization, as witnessed by a range of realworld applications as diverse as bioinformatics [27], community detection in complex networks [19], combinatorial testing [4], FPGA routing [29], planning [30], scheduling [11] and team formation [26], among many others.

In this paper, we investigate how the MaxSAT problem of finitely-valued Łukasiewicz logics can be solved with semantic tableaux. The main objective of our research is to establish the foundations of a generic problem solving paradigm for combinatorial optimization based on Łukasiewicz logic and possibly other many-valued logics complete with respect to a single algebra. We believe that Łukasiewicz logic provides a richer framework than Boolean logic for modeling problems and, in some cases, the resulting encodings may be more natural and compact. Furthermore, extending our results to infinitely-valued logics would allow to solve optimization problems with continuous variables.

Our main contribution is a tableau calculus, with the corresponding proofs of soundness and completeness, for solving the MaxSAT problem of 3-valued Łukasiewicz logic. However, it is important to point out that our approach can be easily extended to other finitely-valued logics complete with respect a single algebra, and, in particular, to any nvalued Łukasiewicz logic.

Complete MaxSAT tableaux calculus for Boolean propositional logical have been defined for clausal [7], [22] and non-clausal formulas [24], as well as cost preserving clausal form transformations for MaxSAT [23].
The paper is structured as follows. Section II defines basic concepts. Section III defines a MaxSAT tableau calculus for 3-valued Łukasiewicz logic and proves its soundness and completeness. Section IV offers the concluding remarks.

\section{PRELIMINARIES}

With the aim of being self-contained, in this section we give a formal definition of many-valued logic and introduce finitely-valued Łukasiewicz logics. For a deeper insight into many-valued logics see e.g. [15].

Definition 1. A propositional language is a pair $\mathbb{L}=\langle\Theta, \alpha\rangle$, where $\Theta$ is a set of logical connectives and $\alpha: \Theta \rightarrow \mathbb{N}$ defines the arity of each connective. Connectives with arity 0 are called constants. A language $\langle\Theta, \alpha\rangle$ with a finite set of connectives $\Theta=\left\{\theta_{1}, \ldots, \theta_{r}\right\}$ is denoted by $\left\langle\theta_{1} / \alpha\left(\theta_{1}\right), \ldots, \theta_{r} / \alpha\left(\theta_{r}\right)\right\rangle$.

Given a set of propositional variables $\mathcal{V}$, the set $L_{\mathcal{V}}$ of $\mathbb{L}$-formulas over $\mathcal{V}$ is inductively defined as the smallest set with the following properties: (i) $\mathcal{V} \subseteq L_{\mathcal{V}}$; (ii) if $\theta \in \Theta$ and $\alpha(\theta)=0$, then $\theta \in L_{\mathcal{V}}$; and (iii) if $\phi_{1}, \ldots, \phi_{m} \in L_{\mathcal{V}}, \theta \in \Theta$ and $\alpha(\theta)=m$, then $\theta\left(\phi_{1}, \ldots, \phi_{m}\right) \in L_{\mathcal{V}}$.

Definition 2. A many-valued logic $\mathcal{L}$ is a tuple $\langle\mathbb{L}, N, A, D\rangle$ where $\mathbb{L}=\langle\Theta, \alpha\rangle$ is a propositional language, $N$ is a truth value set, $A$ is an interpretation of the operation symbols that assigns to each $\theta \in \Theta$ a function $A_{\theta}: N^{\alpha(\theta)} \rightarrow N$ and $D \subset N$ is a set of designated truth values.

The set of designated truth values from the previous definition can be understood as those which affirm satisfaction.

Definition 3. Let $\mathcal{L}$ be a many-valued logic. An interpretation on $\mathcal{L}$ is a function $I: \mathcal{V} \rightarrow N . I$ is extended to arbitrary formulas $\phi$ in the usual way:

1) If $\phi$ is a logical constant, then $I(\phi)=A_{\phi}$.

2) If $\phi=\theta\left(\phi_{1}, \ldots, \phi_{r}\right)$, then $I\left(\theta\left(\phi_{1}, \ldots, \phi_{r}\right)\right)=$ $A_{\theta}\left(I\left(\phi_{1}\right), \ldots, I\left(\phi_{r}\right)\right)$.

A formula $\phi$ is satisfiable iff there is an interpretation $I$ such that $I(\phi) \in D$.

Throughout this work, we focus on a particular family of many-valued logics: the finite-valued Łukasiewicz logics. These were born from the generalization of a three valued logic proposed by J. Łukasiewicz in the early 20th century, the first formal example of a many-valued logic. They 
have been deeply studied since, both from theoretical and practical points of view [14], [17].

The language of Łukasiewicz logic is given by

$$
\mathbb{L}_{\mathrm{Ł} u k}=\langle 0 / 0,1 / 0, \neg / 1, \rightarrow / 2, \wedge / 2, \vee / 2, \odot / 2, \oplus / 2\rangle .
$$

We refer to $\neg$ as negation, $\rightarrow$ as implication, $\wedge$ as weak conjunction, $\vee$ as weak disjunction, $\odot$ as (strong) conjunction, and $\oplus$ as (strong) disjunction.

Definition 4. Given a finite truth value set $N_{n}=$ $\left\{0, \frac{1}{n-1}, \ldots, \frac{n-1}{n-1}\right\}$, the $n$-valued Eukasiewicz logic, denoted by $€_{n}$, is the many-valued logic $\left\langle\mathbb{L}_{€ u k}, N_{n}, A_{€},\{1\}\right\rangle$ where the interpretation of the constants 0,1 is the corresponding values in the algebra, and the interpretation of the operation symbols $A_{€}$ is given by:

$$
\begin{aligned}
A_{\neg}(x) & =1-x \\
A_{\rightarrow}(x, y) & =\min \{1,1-x+y\} \\
A_{\wedge}(x, y) & =\min \{x, y\} \\
A_{\vee}(x, y) & =\max \{x, y\} \\
A_{\odot}(x, y) & =\max \{0, x+y-1\} \\
A_{\oplus}(x, y) & =\min \{1, x+y\}
\end{aligned}
$$

Table I shows the interpretation of the connectives of 3valued Łukasiewicz logic, denoted by $Ł_{3}$, using truth tables. In the following, we will focus our presentation on this logic but our results can be easily adapted to any $n$-valued Łukasiewicz logic.

Table I

TRUTH TABLES FOR 3-VALUED ŁUKASIEWICZ LOGIC ( $\left(Ł_{\mathbf{3}}\right)$

\begin{tabular}{c|ccc}
$\wedge$ & 0 & $\frac{1}{2}$ & 1 \\
\hline 0 & 0 & 0 & 0 \\
$\frac{1}{2}$ & 0 & $\frac{1}{2}$ & $\frac{1}{2}$ \\
1 & 0 & $\frac{1}{2}$ & 1
\end{tabular}

\begin{tabular}{c|ccc}
$\odot$ & 0 & $\frac{1}{2}$ & 1 \\
\hline 0 & 0 & 0 & 0 \\
$\frac{1}{2}$ & 0 & 0 & $\frac{1}{2}$ \\
1 & 0 & $\frac{1}{2}$ & 1
\end{tabular}

\begin{tabular}{c|ccc}
$\vee$ & 0 & $\frac{1}{2}$ & 1 \\
\hline 0 & 0 & $\frac{1}{2}$ & 1 \\
$\frac{1}{2}$ & $\frac{1}{2}$ & $\frac{1}{2}$ & 1 \\
1 & 1 & 1 & 1
\end{tabular}

\begin{tabular}{c|ccc}
$\oplus$ & 0 & $\frac{1}{2}$ & 1 \\
\hline 0 & 0 & $\frac{1}{2}$ & 1 \\
$\frac{1}{2}$ & $\frac{1}{2}$ & 1 & 1 \\
1 & 1 & 1 & 1
\end{tabular}

\begin{tabular}{c|ccccc|c}
$\rightarrow$ & 0 & $\frac{1}{2}$ & 1 & & \\
\hline 0 & 1 & 1 & 1 & & 0 & 1 \\
$\frac{1}{2}$ & $\frac{1}{2}$ & 1 & 1 & & $\frac{1}{2}$ & $\frac{1}{2}$ \\
1 & 0 & $\frac{1}{2}$ & 1 & & 1 & 0
\end{tabular}

Given a Łukasiewicz logic $\mathrm{七}_{n}$, we denote by $S A T^{\mathrm{七}_{n}}$ the set of satisfiable formulas in $Ł_{n}$; i.e.,

$S A T^{\mathfrak{Ł}_{n}}=\left\{\varphi: I(\varphi)=1\right.$ for some interpretation $I$ on $\left.\mathfrak{七}_{n}\right\}$.

The problem of deciding whether or not a formula belongs to the set $S A T^{\mathfrak{Ł}_{n}}$ is called the $\mathbf{L}_{n}$-Satisfiability Problem.
Given a finite multiset of formulas $\Gamma$ of a Łukasiewicz logic $€_{n}$, the problem of finding an interpretation that maximizes the number of satisfied formulas in $\Gamma$ is called the $\mathbf{L}_{n}$ Maximum Satisfiability Problem, or $\mathbf{L}_{n}$-MaxSAT.

Note that we represent MaxSAT instances using multisets instead of sets because repeated formulas cannot be collapsed into one of such formulas as in SAT. In this way, we can keep track of the number of unsatisfied formulas in the input multiset when it is transformed into another multiset that preserves the number of unsatisfied formulas.

In the literature, MaxSAT is also defined as the problem of finding an assignment that maximizes the number of formulas that can be satisfied in a given multiset of formulas. This definition is equivalent to the previous one, because the assignments that maximize the number of satisfied formulas are also the assignments that minimize the number of unsatisfied formulas. We prefer the minimization version because modern MaxSAT solvers find optimal solutions by minimizing the number of unsatisfied formulas.

Since the usual clausal form is not a normal form in Łukasiewicz logic, and even other more complex formulations are not known to be normal forms for this logic (see e.g. [10], [14]), in this paper we study the MaxSAT problem formulated over multisets of formulas, and not clauses.

As in the common approaches to solve the validity and satisfiability problems with tableaux in finitely-valued logics [13], [16], we will use signed formulas [9], defined as follows.

Definition 5. Let $N_{n}=\left\{0, \frac{1}{n-1}, \ldots, \frac{n-1}{n-1}\right\}$ be a finite truth value set and a let $\phi$ be formula of an $n$-valued logic. A signed formula is an expression of the form $\{i\}: \phi$, where $i \in N_{n}$. An interpretation $I$ satisfies $\{i\}: \phi$ iff $I(\phi)=i$.

If $\phi$ is a propositional variable, then $\{i\}: \phi$ is a positive signed literal, and if $\phi$ is a negated propositional variable, then $\{i\}: \phi$ is a negative signed literal.

\section{A MAXSAT TABLEAU CAlCUluS FOR $\succeq_{3}$}

We formally define a MaxSAT tableau calculus for $\iota_{3}$ and prove its soundness and completeness.

Definition 6. A tableau is a tree with a finite number of branches whose nodes are labelled by either a signed propositional formula of $€_{3}$ or a box $(\square)$. A box in a tableau denotes a contradiction. A branch is a maximal path in a tree, and we assume that branches have a finite number of nodes.

Definition 7. Let $\Phi=\left\{\phi_{1}, \ldots, \phi_{m}\right\}$ be a multiset of propositional formulas of $\iota_{3}$. A tableau for $\Phi$ is constructed by a sequence of applications of the following rules:

Initialize A tree with a single branch with $m$ nodes such that each node is labelled with a signed formula of $\Phi=\left\{\{1\}: \phi_{1}, \ldots,\{1\}: \phi_{m}\right\}$ is a tableau for $\Phi$. Such a tableau is called initial tableau and its formulas are declared to be active. 
Table II

MAXSAT TABLEAU RULES FOR LOGIC $\varliminf_{\mathbf{3}}$

\section{weak conjunction}

\begin{tabular}{|c|c|c|c|}
\hline $\multicolumn{2}{|c|}{0}:\left(\phi_{1} \wedge \phi_{2}\right)$ & $\multicolumn{2}{|c|}{1}:\left(\phi_{1} \wedge \phi_{2}\right)$ \\
\hline$\{0\}: \phi_{1}$ & $\begin{array}{l}\mid\{0\}: \phi_{2} \\
\end{array}$ & $\square$ & $\begin{array}{l}\{1\}: \phi_{1} \\
\{1\}: \phi_{2}\end{array}$ \\
\hline $\multicolumn{4}{|c|}{\frac{1}{2}}:\left(\phi_{1} \wedge \phi_{2}\right)$ \\
\hline$\square$ & $\begin{array}{l}\left\{\frac{1}{2}\right\}: \phi_{1} \\
\left\{\frac{1}{2}\right\}: \phi_{2}\end{array}$ & $\begin{array}{l}\left\{\frac{1}{2}\right\}: \phi_{1} \\
\{1\}: \phi_{2}\end{array}$ & $\begin{array}{l}\{1\}: \phi_{1} \\
\left\{\frac{1}{2}\right\}: \phi_{2}\end{array}$ \\
\hline
\end{tabular}

\section{strong conjunction}

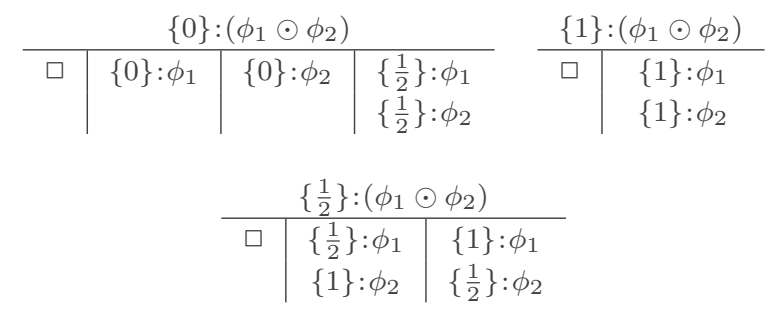

weak disjunction

\begin{tabular}{c|cc|c}
$\multicolumn{2}{l}{0}:\left(\phi_{1} \vee \phi_{2}\right)$ & & $\{1\}:\left(\phi_{1} \vee \phi_{2}\right)$ \\
\hline$\square$ & $\begin{array}{l}\{0\}: \phi_{1} \\
\{0\}: \phi_{2}\end{array}$ & $\{1\}: \phi_{1} \mid$ & $\{1\}: \phi_{2}$ \\
& $\multicolumn{3}{c}{\frac{1}{2}}:\left(\phi_{1} \vee \phi_{2}\right)$ \\
\hline$\square$ & $\{0\}: \phi_{1}$ & $\left\{\frac{1}{2}\right\}: \phi_{1}$ & $\left\{\frac{1}{2}\right\}: \phi_{1}$ \\
& $\left\{\frac{1}{2}\right\}: \phi_{2}$ & $\left\{\frac{1}{2}\right\}: \phi_{2}$ & $\{0\}: \phi_{2}$
\end{tabular}

strong disjunction

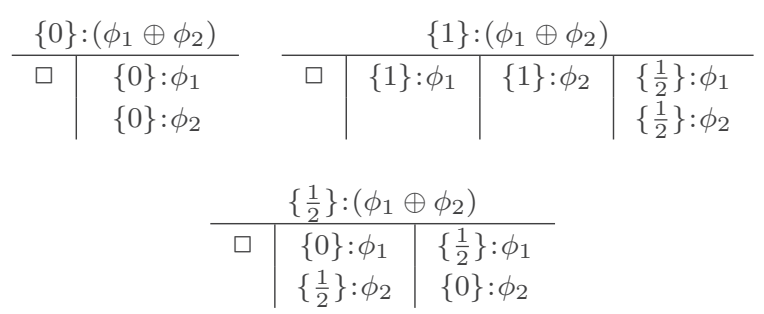

\section{implication}

\begin{tabular}{|c|c|c|c|c|c|c|c|}
\hline $\multicolumn{3}{|c|}{0}:\left(\phi_{1} \rightarrow \phi_{2}\right)$ & $\multicolumn{5}{|c|}{1}:\left(\phi_{1} \rightarrow \phi_{2}\right)$ \\
\hline \multirow[t]{8}{*}{$\square$} & \multirow{6}{*}{\multicolumn{2}{|c|}{$\begin{array}{l}\{1\}: \phi_{1} \\
\{0\}: \phi_{2}\end{array}$}} & $\square$ & & $\phi_{1}$ & $\{1\}: \phi_{2}$ & $\left\{\frac{1}{2}\right\}: \phi_{1}$ \\
\hline & & & & & & & $\left\{\frac{1}{2}\right\}: \phi_{2}$ \\
\hline & & & $\multicolumn{4}{|c|}{\frac{1}{2}}:\left(\phi_{1} \rightarrow \phi_{2}\right)$ & \\
\hline & & & \multirow{2}{*}{\multicolumn{4}{|c|}{\begin{tabular}{l|l}
$\left\{\frac{1}{2}\right\}: \phi_{1}$ & $\{1\}: \phi_{1}$ \\
$\{0\}: \phi_{2}$ & $\left\{\frac{1}{2}\right\}: \phi_{2}$
\end{tabular}}} & \\
\hline & & & & & & & \\
\hline & & & \multicolumn{4}{|c|}{ negation } & \\
\hline & $\multicolumn{2}{|c|}{0}: \neg \phi$ & $\multicolumn{3}{|c|}{\frac{1}{2}}: \neg \phi$ & $\multicolumn{2}{|l|}{1}: \neg \phi$ \\
\hline & $\multicolumn{2}{|c|}{1}: \phi$ & $\multicolumn{3}{|c|}{\frac{1}{2}}: \phi$ & $\multicolumn{2}{|l|}{0}: \phi$ \\
\hline
\end{tabular}

Expansion Given a tableau $T$ for $\Phi$, a branch $b$ of $T$, and a node of $b$ labelled with an active signed formula $\{i\}: F$, where $i \in\left\{0, \frac{1}{2}, 1\right\}$, whose main connective is in $\{\neg, \rightarrow, \wedge, \vee, \odot, \oplus\}$, we first identify the expansion rule of Table II whose premise matches with $\{i\}: F$ and then append as many new branches as extensions the rule has and as many nodes per branch as formulas in each extension. The derived tableau is a tableau for $\Phi$, formula $\{i\}: F$ becomes inactive in $b$ and the added signed formulas are declared to be active.

Derivation of contradictions Given a tableau $T$ for $\Phi$, a branch $b$ of $T$ in which the only active formulas are positive signed literals, the multiset $L$ of positive signed literals of $b$ containing the variable $x$, and the list $j_{1}, j_{2}, \ldots, j_{k}(k \leq 3)$ of truth values occurring in $L$ sorted in decreasing order of the number of occurrences in $L$, the tableau obtained by appending a node below $b$ labelled with $\square$ for each signed literal of the form $\left\{j_{2}\right\}: x, \ldots,\left\{j_{k}\right\}: x$ is a tableau for $\Phi$, and such literals become inactive in $b$.

Table II displays the expansion rules of the proposed tableau calculus for $£_{3}$. Note that all the rules preserve the number of premises falsified by an assignment in at least one branch and do not decrease that number in the other branches (if any). Observe that there are certain extensions of the rules of binary connectives that just contain one box; this is to avoid to assign more than one contradiction to a formula whose left and right sub-formulas are inconsistent, thus preserving the number of unsatisfied formulas at the upper most level. Note also that we add the sign $\{1\}$ to the input formulas when we build the initial tableau; this is because 1 is the designated truth value of $Ł_{3}$.

The goal of the described derivation of contradictions is to minimize the number of detected contradictions. When tableaux are used to determine satisfiability or validity in many-valued logics, a contradiction is derived as soon as a branch contains two literals $\{i\}: x$ and $\{j\}: x$ such that $i \neq j$. However, this approach is unsound in manyvalued MaxSAT. For example, it is clear that the minimum number of unsatisfied positive signed literals in the multiset $\left\{\{0\}: x,\left\{\frac{1}{2}\right\}: x,\{1\}: x,\{1\}: x\right\}$ is 2 . However, for instance if the previous positive signed literals occur in that order in a tableau branch and we apply the usual contradiction rule (derive a contradiction from $\{i\}: x$ and $\{j\}: x$ if $i \neq j$ as soon as the signed literals appear, and mark those points in the tableau), we derive only one contradiction, since once the signed literals $\{0\}: x,\left\{\frac{1}{2}\right\}: x$ are marked, no further contradiction would arise.

The new rule for deriving contradictions takes the most common value assigned to a variable in a branch (in order to minimize the number of contradictions), and adds a contradiction $(\square)$ for each other point in the branch assigning a different value to the same variable. For that reason (i.e., 
Figure 1. A tableau for solving the MaxSAT instance $\left\{\neg x_{1}, \neg x_{2}, x_{1} \odot\right.$ $\left.x_{2}, x_{1} \vee x_{2}\right\}$.

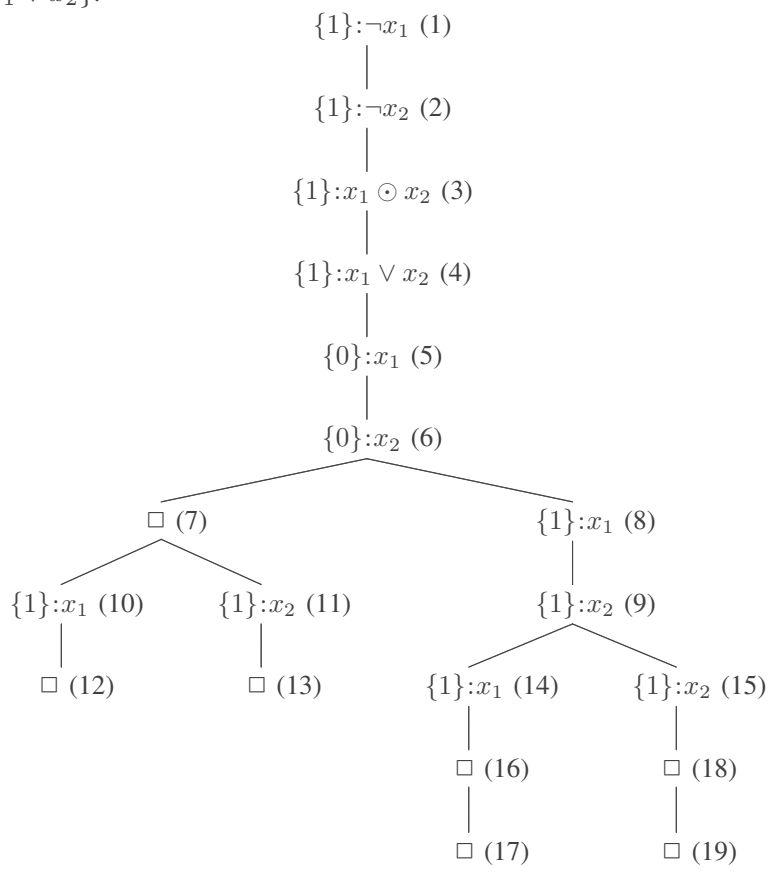

it is necessary to know the most-common assignment for a variable in a branch), the rule needs to be applied after all the complex formulas have been expanded to the (signed) variable level.

Definition 8. Let $T$ be a tableau for a multiset of propositional formulas $\Phi$ of $\varkappa_{\mathbf{3}}$. A branch $b$ of $T$ is saturated when no further expansion rules can be applied and no further contradictions can be derived on $b$, and $T$ is completed when all its branches are saturated. The cost of a saturated branch is the number of derived contradictions on the branch. The cost of a completed tableau is the minimum cost among all its branches.

As we show below, the minimum number of formulas that can be unsatisfied in a multiset of propositional formulas $\Phi$ of $\iota_{3}$ is $k$ iff the cost of a completed tableau for $\Phi$ is $k$. Thus, the systematic construction of a completed tableau for $\phi$ provides an exact method for solving the MaxSAT problem of $\iota_{3}$.

Example 1. We can determine the minimum number of unsatisfied formulas in the multiset $\Phi=\left\{\neg x_{1}, \neg x_{2}, x_{1} \odot\right.$ $\left.x_{2}, x_{1} \vee x_{2}\right\}$ using the previous tableau calculus. Figure 1 displays how the tableau is constructed. The numbers between parentheses do not belong to the tableau; they are used to refer to the nodes. We start by constructing the initial tableau (1-4) and apply the expansion rule of negation to (1), obtaining (5), and to (2), obtaining (6). We then apply the expansion rule of strong conjunction to (3), obtaining
(7) and (8-9). On the left branch, we first apply the the expansion rule of weak disjunction to (4), obtaining (10) and (11) and then derive the contradictions (12) and (13). On the right branch, we first apply the the expansion rule of weak disjunction to (4), obtaining (14) and (15) and then derive the contradictions (16-17) and (18-19). Note that now each branch is saturated and the tableau is completed. Since the minimum number of boxes among the branches of the completed tableau is 2 , the minimum number of formulas that can be unsatisfied in $\Phi$ is 2 .

\section{A. Soundness and completeness}

In this section we prove the soundness and completeness of the proposed MaxSAT tableau calculus for $\iota_{3}$. We first prove two propositions needed later.

Proposition 9. A tableau for a multiset of propositional formulas $\Phi$ of $\iota_{3}$ is completed in a finite number of steps.

Proof: We start by creating an initial tableau and then apply expansion rules in the newly created branches until saturation. The rules reduce the number of connectives. Since we began with a finite number of connectives, these rules can only be applied a finite number of times. The derivation of contradictions after applying the expansion rules is also finite because there are a finite number of literals and the literals used to derive a contradiction become inactive. Hence, the construction of any completed tableau terminates in a finite number of steps.

Proposition 10. An assignment I falsifies $k$ premises of an expansion rule iff assignment I falsifies $k$ conclusions in one branch of the conclusions of the rule and at least $k$ conclusions in the other branches (if any).

Proof: We should prove the result for each rule. Due to the lack of space we prove the proposition for the expansion rule of weak conjunction. The other rules are proven to be sound in the same fashion. We distinguish three cases:

- The premise is $\{0\}:\left(\phi_{1} \wedge \phi_{2}\right)$. By inspecting the truth table we see that $\{0\}:\left(\phi_{1} \wedge \phi_{2}\right)$ is satisfied iff $\phi_{1}$ or $\phi_{2}$ is assigned 0 . Hence, if $\{0\}:\left(\phi_{1} \wedge \phi_{2}\right)$ is unsatisfied both extensions are unsatisfied too, and the number of unsatisfied formulas is preserved; if $\{0\}:\left(\phi_{1} \wedge \phi_{2}\right)$ is satisfied, at least one extension is satisfied.

- The premise is $\left\{\frac{1}{2}\right\}:\left(\phi_{1} \wedge \phi_{2}\right)$. By inspecting the truth table we see that $\phi_{1} \wedge \phi_{2}$ is assigned the truth value $\frac{1}{2}$ when $\phi_{1}$ and $\phi_{2}$ are both assigned $\frac{1}{2}$ or when $\phi_{1}$ and $\phi_{2}$ are assigned $\frac{1}{2}(1)$ and $1\left(\frac{1}{2}\right)$. In these cases, at least one extension is satisfied. In the rest of cases, all the extensions are unsatisfied. The rule has an extension with a single box to avoid to count two contradictions when an extension contains two unsatisfied formulas.

- The premise is $\{1\}:\left(\phi_{1} \wedge \phi_{2}\right)$. By inspecting the truth table we see that $\phi_{1} \wedge \phi_{2}$ is assigned the truth value 1 iff $\phi_{1}$ and $\phi_{2}$ are both assigned 1. In this case, the 
right extension is satisfied. In the rest of cases, all the extensions are unsatisfied and there is an extension with a single box to avoid to count two contradictions when the two formulas of the right extension are unsatisfied.

Theorem 11. Soundness \& completeness. The cost of $a$ completed tableau for a multiset of formulas $\Phi$ is $m$ iff the minimum number of unsatisfied formulas in $\Phi$ is $m$.

Proof: (Soundness:) Assume a tableau $T$ for $\Phi$ was obtained by creating a sequence of tableaux $T_{0}, \ldots, T_{n}$ $(n \geq 0)$ such that $T_{0}$ is an initial tableau for $\Phi, T_{n}=T$, and $T_{i}$ was obtained by a single application of an expansion rule or by deriving contradictions on an branch of $T_{i-1}$ for $i=1, \ldots, n$. By Proposition 9, we know that such a sequence is finite. Since $T$ has cost $m, T_{n}$ contains one branch $b$ with exactly $m$ boxes and the rest of branches contain at least $m$ boxes. Moreover, the active formulas in the branches of $T_{n}$ are positive signed literals that contain the same truth value for the same variable. The assignment that sets the value of its sign to the variable of each active literal in $b$, only falsifies the $m$ boxes and there cannot be any assignment satisfying less than $m$ formulas in a branch of $T_{n}$ because each branch contains at least $m$ boxes. Therefore, the minimum number of active formulas than can be unsatisfied among the branches of $T_{n}$ is $m$.

Proposition 10 guarantees that the minimum number of unsatisfied active formulas is preserved in the sequence of tableaux $T_{0}, \ldots, T_{n}$. Thus, the minimum number of unsatisfied active formulas in $T_{0}$ is also $m$. Since $T_{0}$ is formed by a single branch that only contains the formulas in $\Phi$ and all these formulas are active, the minimum number of formulas that can be unsatisfied in $\Phi$ is $m$.

(Completeness:) Assume that there is a completed tableau $T$ for $\Phi$ that does not have cost $m$. We distinguish two cases:

(i) $T$ has a branch $b$ of cost $k$, where $k<m$. Then, $T$ has a branch with $k$ boxes and a satisfiable multiset of signed literals because $T$ is completed. This implies that the minimum number of unsatisfied active formulas among the branches of $T$ is at most $k$. By Proposition 10, this also holds for $T_{0}$, but this is in contradiction with $m$ being the minimum number of unsatisfied formulas in $\Phi$ because $k<m$. Thus, any branch of $T$ has at least cost $m$.

(ii) $T$ has no branch of cost $m$. This is in contradiction with $m$ being the minimum number of unsatisfied formulas in $\Phi$. Since the tableau rules preserve the minimum number of unsatisfied formulas and the branches of any completed tableau only contain active formulas that are boxes or positive signed literals, $T$ must have a saturated branch with $m$ boxes. Thus, $T$ has a branch of cost $m$.

Hence, each completed tableau $T$ for a multiset of formulas $\Phi$ has cost $m$ if the minimum number of formulas that can be unsatisfied in $\Phi$ is $m$.

\section{CONCLUDING REMARKS}

We defined a complete tableau calculus for MaxSAT over 3-valued Łukasiewicz logic, and proved that it is sound and complete. The proposed approach establishes the foundations of a generic problem solving paradigm for combinatorial optimization based on Łukasiewicz logic.

That approach can be generalized to other finitely-valued logics arising from a single algebra, and, in particular, to any $n$-valued Łukasiewicz logic. In this case, we should define a tableau expansion rule for each truth value and each connective in such a way that the expansion rules defined for each connective reflect the semantic information of the corresponding truth table as we did for 3-valued Łukasiewicz logic.

As future work we plan to find combinatorial optimization problems that admit more natural encodings using Łukasiewicz logic and empirically compare the obtained many-valued approach with the Boolean approach. We also plan to study the infinitely-valued case, that would allow to address MaxSAT questions in a continuous environment.

\section{ACKNOWLEDGMENT}

This work was supported by MINECO-FEDER project RASO TIN2015-71799-C2-1-P.

\section{REFERENCES}

[1] A. Abramé and D. Habet, "On the resiliency of unit propagation to Max-Resolution," in Proceedings of the 24th International Joint Conference on Artificial Intelligence, IJCAI, Buenos Aires, Argentina, 2015, pp. 268-274.

[2] T. Alsinet, F. Manyà, and J. Planes, "A Max-SAT solver with lazy data structures," in Proceedings of the 9th IberoAmerican Conference on Artificial Intelligence, IBERAMIA 2004, Puebla, México. Springer LNCS 3315, 2004, pp. 334342.

[3] C. Ansótegui, M. L. Bonet, and J. Levy, "SAT-based MaxSAT algorithms," Artificial Intelligence, vol. 196, pp. 77-105, 2013.

[4] C. Ansótegui, I. Izquierdo, F. Manyà, and J. T. Jiménez, "A Max-SAT-based approach to constructing optimal covering arrays," in Proceedings of the 16th International Conference of the Catalan Association for Artificial Intelligence, CCIA 2013, Vic, Spain, ser. Frontiers in Artificial Intelligence and Applications, vol. 256. IOS Press, 2013, pp. 51-59.

[5] J. Argelich, A. Cabiscol, I. Lynce, and F. Manyà, "Efficient encodings from CSP into SAT, and from MaxCSP into MaxSAT," Journal of Multiple-Valued Logic and Soft Computing, vol. 19, no. 1-3, pp. 3-23, 2012.

[6] J. Argelich, C. M. Li, F. Manyà, and J. Planes, "The first and second Max-SAT evaluations," Journal on Satisfiability, Boolean Modeling and Computation, vol. 4, pp. 251-278, 2008. 
[7] J. Argelich, C. M. Li, F. Manyà, and J. R. Soler, "Clause tableaux for maximum and minimum satisfiability," Logic Journal of IGPL, 2020.

[8] J. Argelich and F. Manyà, "Exact Max-SAT solvers for overconstrained problems," Journal of Heuristics, vol. 12, no. 4-5, pp. 375-392, 2006.

[9] B. Beckert, R. Hähnle, and F. Manyà, "The SAT problem of signed CNF formulas," in Labelled Deduction, ser. Applied Logic Series, D. Basin, M. D'Agostino, D. Gabbay, S. Matthews, and L. Viganò, Eds. Kluwer, Dordrecht, 2000, vol. 17 , pp. 61-82.

[10] M. Bofill, F. Manyà, A. Vidal, and M. Villaret, "New complexity results for Łukasiewicz logic," Soft Computing, vol. 23, no. 7, pp. 2187-2197, 2019.

[11] M. Bofill, M. Garcia, J. Suy, and M. Villaret, "MaxSATbased scheduling of B2B meetings," in Proceedings of the 12th International Conference on Integration of AI and OR Techniques in Constraint Programming, CPAIOR, Barcelona, Spain, 2015, pp. 65-73.

[12] M. L. Bonet, J. Levy, and F. Manyà, "Resolution for MaxSAT," Artificial Intelligence, vol. 171, no. 8-9, pp. 240-251, 2007.

[13] W. A. Carnielli, "Systematization of finite many-valued logics through the method of tableaux," Journal of Symbolic Logic, vol. 52, no. 2, pp. 473-493, 1987.

[14] R. Cignoli, I. M. L. D'Ottaviano, and D. Mundici, Algebraic foundations of many-valued reasoning, ser. Trends in Logic-Studia Logica Library. Dordrecht: Kluwer Academic Publishers, 2000, vol. 7. [Online]. Available: http://books.google.es/books?id=QFcrAAAAYAAJ

[15] P. Cintula, P. Hájek, and C. Noguera, Eds., Handbook of Mathematical Fuzzy Logic, 2 volumes, ser. Studies in Logic. Mathematical Logic and Foundation. College Publications, 2011, vol. 37 and 38 .

[16] R. Hähnle, Automated Deduction in Multiple-Valued Logics, ser. International Series of Monographs in Computer Science. Oxford University Press, 1994, vol. 10.

[17] P. Hájek, Metamathematics of Fuzzy Logic. Kluwer, Dordrecht, 1998.

[18] A. Ignatiev, A. Morgado, and J. Marques-Silva, "RC2: an efficient maxsat solver," J. Satisf. Boolean Model. Comput., vol. 11, no. 1, pp. 53-64, 2019.

[19] S. Jabbour, N. Mhadhbi, B. Raddaoui, and L. Sais, "A SATbased framework for overlapping community detection in networks," in Proceedings of the 21st Pacific-Asia Conference on Advances in Knowledge Discovery and Data Mining, Part II, PAKDD, Jeju, South Korea, 2017, pp. 786-798.

[20] C. M. Li and F. Manyà, "MaxSAT, hard and soft constraints," in Handbook of Satisfiability, A. Biere, H. van Maaren, and T. Walsh, Eds. IOS Press, 2009, pp. 613-631.
[21] C. M. Li, F. Manyà, N. O. Mohamedou, and J. Planes, "Resolution-based lower bounds in MaxSAT," Constraints, vol. 15 , no. 4, pp. 456-484, 2010.

[22] C. M. Li, F. Manyà, and J. R. Soler, "A clause tableaux calculus for MaxSAT," in Proceedings of the 25th International Joint Conference on Artificial Intelligence, IJCAI-2016, New York, USA, 2016, pp. 766-772.

[23] C. M. Li, F. Manyà, and J. R. Soler, "Clausal form transformation in MaxSAT," in Proceedings, 49th International Symposium on Multiple-Valued Logics (ISMVL), Fredericton, NB, Canada. IEEE CS Press, 2019, pp. 30-35.

[24] _ - "A tableau calculus for non-clausal maximum satisfiability," in Proceedings of the 28th International Conference on Automated Reasoning with Analytic Tableaux and Related Methods, TABLEAUX, London, UK. Springer LNCS 11714, 2019, pp. 58-73.

[25] I. Lynce, V. M. Manquinho, and R. Martins, "Parallel maximum satisfiability," in Handbook of Parallel Constraint Reasoning, Y. Hamadi and L. Sais, Eds. Springer, 2018, pp. 61-99.

[26] F. Manyà, S. Negrete, C. Roig, and J. R. Soler, "A MaxSATbased approach to the team composition problem in a classroom," in Autonomous Agents and Multiagent Systems - AAMAS 2017 Workshops, Visionary Papers, São Paulo, Brazil, Revised Selected Papers. Springer LNCS 10643, 2017, pp. 164-173.

[27] J. Marques-Silva, J. Argelich, A. Graça, and I. Lynce, "Boolean lexicographic optimization: algorithms \& applications," Annals of Matematics and Artificial Intelligence, vol. 62, no. 3-4, pp. 317-343, 2011.

[28] N. Narodytska and F. Bacchus, "Maximum satisfiability using core-guided MaxSAT resolution," in Proceedings of the 28th AAAI Conference on Artificial Intelligence, Québec City, Canada., 2014, pp. 2717-2723.

[29] H. Xu, R. A. Rutenbar, and K. A. Sakallah, "sub-SAT: a formulation for relaxed boolean satisfiability with applications in routing," IEEE Trans. on CAD of Integrated Circuits and Systems, vol. 22, no. 6, pp. 814-820, 2003.

[30] L. Zhang and F. Bacchus, "MAXSAT heuristics for cost optimal planning," in Proceedings of the 26th AAAI Conference on Artificial Intelligence, Toronto, Ontario, Canada, 2012, pp. 1846-1852. 